\title{
Inverse Task of Vibration Active Reduction of Mechanical Systems
}

\author{
Andrzej Dymarek and Tomasz Dzitkowski \\ Institute of Engineering Processes Automation and Integrated Manufacturing Systems, \\ Silesian University of Technology, Konarskiego 18A, 44-100 Gliwice, Poland \\ Correspondence should be addressed to Andrzej Dymarek; andrzej.dymarek@polsl.pl
}

Received 11 April 2016; Revised 4 September 2016; Accepted 21 September 2016

Academic Editor: Tzu-Yang Yu

Copyright ( 2016 A. Dymarek and T. Dzitkowski. This is an open access article distributed under the Creative Commons Attribution License, which permits unrestricted use, distribution, and reproduction in any medium, provided the original work is properly cited.

\begin{abstract}
The paper presents the problem of discrete vibration reduction in mechanical systems depending on the desired dynamic properties. The conditions for physical feasibility of dynamic characteristics have been defined, in the form of impedance and mobility, for passive and active vibration reduction. The authors have presented a graphic method for determining the free vibration drop coefficient, based on the desired value of the reduced resonance frequency amplitude.
\end{abstract}

\section{Introduction}

The reduction of vibration is related with determining, at a given point of a system, the force with parameters that allow controlling the vibration amplitude of a chosen point in the feedback loop [1-6], or utilization in the system socalled energy dissipating components [3, 4, 7-9]. The values of active and passive components are generated, in systems subjected to vibration reduction, on the basis of information on the dynamic state of the system. The conditions, under which the structural and parametric identification of the system subject to vibration reduction is realized, should meet in advance the required dynamic properties $[2,6,8-10]$.

The synthesis, as the inverse task, enables the identification of parameters for the desired dynamic properties in the form of resonance frequencies and amplitude values. In such cases, it is therefore necessary to specify the criterion for searching the structure and parameters of the model based on knowledge of the dynamic properties of the real object.

The paper concerns the formulation and solution the problem of active vibration reduction of discrete mechanical systems in view of the desired dynamic properties $[2-4,7,11-$ 13]. For this purpose, it has formulated the conditions of physical realisability of dynamic characteristics in the form of a impedance and mobility in the case of passive and active vibration reduction. It also presented the graphical method for determining the damping coefficient based on the desired value of the amplitude of the reduced resonance frequency. Moreover, it has proposed the method of searching for the active force that reduces vibration to the desired amplitude value.

\section{Synthesis: The Inverse Problem of Vibrating Systems}

One of the conditions of assuming the proper model of a machine, at the stage of structural and parametric identification of the object, is the synthesis of mechanical systems with the desired properties [14-16]. Synthesis or the inverse problem of vibrating systems is understood in this work as a search for the structure parameters based on the known dynamic properties [17-20].

2.1. Dynamic Characteristics and Properties of the Identified System. The characteristics of the input-output (forcevelocity) relationships, subjected to the direct identification with the methods of the synthesis of a discrete vibrating system, are dynamic function in the form of the mechanical impedance $Z(s)$ or the mobility $Y(s)$, describing in unambiguously way its state [21-24]. The dynamic characteristics, subjected to the identification process with synthesis 
methods, are positive real-valued rational functions of the following forms:

$$
\begin{aligned}
& Y(s)=\frac{v}{f}=\frac{1}{Z(s)}=\frac{d_{l} s^{l}+d_{l-1} s^{l-1}+\cdots+d_{0}}{c_{k} s^{k}+c_{k-1} s^{k-1}+\cdots+c_{0}}, \\
& \quad l=k+1, \\
& Z(s)=\frac{f}{v}=\frac{1}{Y(s)}=\frac{c_{k} s^{k}+c_{k-1} s^{k-1}+\cdots+c_{0}}{d_{l} s^{l}+d_{l-1} s^{l-1}+\cdots+d_{0}}, \\
& k=l+1,
\end{aligned}
$$

where $k, l$ are natural numbers, $v$ is velocity, $f$ is force, $s$ is Laplace operator, and $d_{l}, \ldots, d_{0}, c_{k}, \ldots, c_{0}$ are real numbers.

Generally, the synthesis of vibrating mechanical systems comes down to the prime factorization of the characteristic function (decomposition into partial fractions, decomposition into continued fractions). In this work, the mixed method formalized by the authors in [20] is used.

Prior to the synthesis with the mixed method, the characteristic function of the mobility $Y_{U}(s)$ or the mechanical impedance $Z_{U}(s)$ should be designated. For this purpose, on the basis of the desired dynamic properties in the form of a sequence of resonance frequencies $\omega_{p 1}, \omega_{p 2}, \ldots, \omega_{p n}$ and antiresonance frequencies $\omega_{z 1}, \omega_{z 2}, \ldots, \omega_{z n}$ which are, respectively, the poles and zeros of the searched characteristics $Y_{U}(s)$, the admittance function $V_{U}(s)$ or the dynamic stiffness $U_{U}(s)$ is determined in the form

$$
\begin{aligned}
& U_{U}(s)=\frac{1}{V_{U}(s)}=H \frac{\prod_{i=1}^{n}\left(s^{2}+\omega_{p i}^{2}\right)}{\prod_{j=1}^{n-1}\left(s^{2}+\omega_{z j}^{2}\right)}=H \frac{L(s)}{M(s)}, \\
& \quad i, j=1,2,3, \ldots, n, \\
& \omega_{p 1}<\omega_{z 1}<\omega_{p 2}<\omega_{z 2} \cdots \omega_{p n}<\omega_{z n},
\end{aligned}
$$

where $\omega_{p 1}, \omega_{p 2}, \ldots, \omega_{p n}$ are resonance frequencies, the pools of the function $V_{U}(s), H$ is proportionality constant, and $\omega_{z 1}, \omega_{z 2}, \ldots, \omega_{z n}$ are antiresonance frequencies, the zeros of the function $V_{U}(s)$.

Characteristic functions of the mobility $Y_{U}(s)$ or the impedance $Z_{U}(s)$, directly subjected to the synthesis method, are determined using the transformation in relation to the admittance $V_{U}(s)$ or the stiffness $U_{U}(s)$ in the form

$$
\begin{gathered}
Y_{U}(s)=s V_{U}(s), \\
Z_{U}(s)=\frac{1}{s} U_{U}(s),
\end{gathered}
$$

where $Y_{U}(s)$ is dynamic mobility, $V_{U}(s)$ is admittance, $s$ is variable determined as the Laplace operator, described by the formula $s=i \omega, Z_{U}(s)$ is mechanical impedance, and $U_{U}(s)$ is dynamic stiffness.

The resulting functions transform the motion description of the system from generalized displacements $x$ and generalized forces $f\left(V_{U}(s)=1 / U_{U}(s)=x / f\right)$ to the motion description using generalized velocities $v$ and generalized forces $f\left(Y_{U}(s)=1 / Z_{U}(s)=v / f\right)$, fulfilling the condition $|k-l|=1$ as in the forms in (1).
2.2. Method of Mixed Synthesis. The mixed method of synthesis of vibrating systems is based on the distribution of the characteristic function, in the form $Y_{U}(s)$ or $Z_{U}(s)$ on the sum of rational functions, the number of which depends on the adopted resonance frequencies. In extreme cases, it is the sum of partial fractions or a continued fraction of the analyzed dynamic characteristic $Y_{U}(s)$ or $Z_{U}(s)$ describing the dynamic properties. Due to the nature of the distribution of the considered dynamic characteristics, the presented method of synthesis could be treated as generalization of the known methods of synthesis, not only in the field of mechanics, but also in the field of electric engineering of systems with concentrated parameters. To these methods are included the Foster method (expansion of the dynamic characteristics into a continued fraction CFE) and the Cauer method (expansion of the characteristics into partial fractions PFE). Beginning the formalization of the presented method, it should be assumed that the dynamic properties of the required or being identified system are given. These properties are given in the form of known resonance $\left(\omega_{p 1}, \omega_{p 2}, \ldots, \omega_{p n}\right)$ and antiresonance frequencies $\left(\omega_{z 1}, \omega_{z 2}, \ldots, \omega_{z n}\right)$, on the basis of which the dynamic characteristics $Y_{U}(s)$ or $Z_{U}(s)$ subjected to the synthesis with the mixed method are determined. In the general case, the function $Z_{U}(s)$, of systems on which there are imposed constraints, takes the form

$$
\begin{aligned}
Z_{U}(s) & =\frac{U_{U}(s)}{s}=\frac{1}{s V_{U}(s)}=H \frac{\prod_{i=1}^{n}\left(s^{2}+\omega_{p i}^{2}\right)}{s \prod_{j=1}^{n-1}\left(s^{2}+\omega_{z j}^{2}\right)} \\
& =H \frac{L(s)}{M(s)}, \quad i, j=1,2,3, \ldots, n . \\
\omega_{p 1} & <\omega_{z 1}<\omega_{p 2}<\omega_{z 2} \cdots \omega_{p n}<\omega_{z n},
\end{aligned}
$$

where $\omega_{p 1}, \omega_{p 2}, \ldots, \omega_{p n}$ are resonance frequencies, the pools of the mobility function $Y_{U}(s)$, and $\omega_{z 1}, \omega_{z 2}, \ldots, \omega_{z n}$ are antiresonance frequencies, the zeros of the mobility function $Y_{U}(s)$.

The determined dynamic characteristic $Z_{U}(s)=1 / Y_{U}(s)$ is decomposed into partial fractions, obtaining

$$
\begin{aligned}
\frac{Z_{U}(s)}{H}= & k_{\infty} s+\frac{k_{0}}{s}+\frac{A_{1}}{\left(s-i \omega_{z 1}\right)}+\frac{A_{2}}{\left(s+i \omega_{z 1}\right)}+\cdots \\
& +\frac{A_{2 n-1}}{\left(s-i \omega_{z n}\right)}+\frac{A_{2 n}}{\left(s+i \omega_{z n}\right)}
\end{aligned}
$$

where $k_{\infty}, k_{0}, A_{1}, A_{2}, \ldots, A_{2 n-1}, A_{2 n}$ are values of residues in pools, equal, respectively, to $\infty, i \omega_{z 1},-i \omega_{z 1}, \ldots, i \omega_{z n},-i \omega_{z n}$, $i=\sqrt{-1}$.

Values of residues $k_{\infty}, k_{0}, A_{1}, A_{2}, \ldots, A_{2 n-1}, A_{2 n}$ are determined using

$$
\begin{aligned}
& k_{\infty}=\lim _{s \rightarrow \infty} \frac{Z_{U}(s)}{s} \\
& k_{0}=\lim _{s \rightarrow 0} s Z_{U}(s)
\end{aligned}
$$




$$
\begin{gathered}
A_{1}=\lim _{s \rightarrow i \omega_{z a}}\left(s-i \omega_{z 1}\right) Z_{U}(s), \\
A_{2}=\lim _{s \rightarrow-i \omega_{z a}}\left(s+i \omega_{z 1}\right) Z_{U}(s) \\
\vdots \\
A_{2 n-1}=\lim _{s \rightarrow i \omega_{z n}}\left(s-i \omega_{z n}\right) Z_{U}(s), \\
A_{2 n}=\lim _{s \rightarrow-i \omega_{z n}}\left(s+i \omega_{z n}\right) Z_{U}(s) .
\end{gathered}
$$

Function equation (4) is a positive real-valued rational function, which means that all residues on the imaginary axis are real positive numbers, and $A_{1}, A_{2}, \ldots, A_{2 n-1}, A_{2 n}$ are the values of residues of conjugate numbers. It could be saved as

$$
A_{1}=A_{2}=k_{1}, \ldots, A_{2 n-1}=A_{2 n}=k_{n}
$$

and hence

$$
\begin{gathered}
\frac{A_{1}}{\left(s-i \omega_{z 1}\right)}+\frac{A_{2}}{\left(s+i \omega_{z 1}\right)}=\frac{2 k_{1} s}{\left(s^{2}+\omega_{z 1}^{2}\right)} \\
\frac{A_{3}}{\left(s-i \omega_{z 2}\right)}+\frac{A_{4}}{\left(s+i \omega_{z 2}\right)}=\frac{2 k_{2} s}{\left(s^{2}+\omega_{z 2}^{2}\right)} \\
\frac{A_{5}}{\left(s-i \omega_{z 3}\right)}+\frac{A_{6}}{\left(s+i \omega_{z 3}\right)}=\frac{2 k_{3} s}{\left(s^{2}+\omega_{z 3}^{2}\right)} \\
\vdots \\
\frac{A_{2 n-1}}{\left(s-i \omega_{z n}\right)}+\frac{A_{2 n}}{\left(s+i \omega_{z n}\right)}=\frac{2 k_{n} s}{\left(s^{2}+\omega_{z n}^{2}\right)} .
\end{gathered}
$$

Finally, the distribution of impedance function equation (4) into partial fractions taking into account (9) in (6) takes the form

$$
\begin{aligned}
\frac{Z_{U}(s)}{H}= & k_{\infty} s+\frac{k_{0}}{s}+\frac{2 k_{1} s}{\left(s^{2}+\omega_{z 1}^{2}\right)}+\frac{2 k_{2} s}{\left(s^{2}+\omega_{z 2}^{2}\right)} \\
& +\frac{2 k_{3} s}{\left(s^{2}+\omega_{z 3}^{2}\right)}+\cdots+\frac{2 k_{(n-1)} s}{\left(s^{2}+\omega_{z(n-1)}^{2}\right)}
\end{aligned}
$$

Such obtained mechanical impedance $Z_{U}(s)$, in the form of "partial fractions," could be represented as the sum of any rational functions. Through the addition of the selected components of function equation (10), whose number depends on the adopted antiresonance frequencies, impedance equation (4) is determined in one of the following forms:

$$
\begin{aligned}
\frac{Z_{U}(s)}{H}= & k_{\infty} s+\frac{k_{0}}{s}+\frac{2 k_{1} s}{\left(s^{2}+\omega_{z 1}^{2}\right)}+\cdots \\
& +\frac{B_{1} s^{2 n-3}+B_{2} s^{2 n-5}+\cdots+B_{0} s}{\left(s^{2}+\omega_{z 2}^{2}\right)\left(s^{2}+\omega_{z 3}^{2}\right) \cdots\left(s^{2}+\omega_{z(n-1)}^{2}\right)}
\end{aligned}
$$

$$
\begin{aligned}
\frac{Z_{U}(s)}{H}= & k_{\infty} s+\frac{k_{0}}{s}+\frac{2 k_{1} s}{\left(s^{2}+\omega_{z 1}^{2}\right)}+\cdots+\frac{2 k_{2} s}{\left(s^{2}+\omega_{z 2}^{2}\right)} \\
& +\frac{C_{1} s^{2 n-6}+C_{2} s^{2 n-8}+\cdots+C_{0} s}{\left(s^{2}+\omega_{z 3}^{2}\right) \cdots\left(s^{2}+\omega_{z(n-1)}^{2}\right)} \\
\frac{Z_{U}(s)}{H}= & \sum_{i=1}^{n} k_{i \infty} s+\frac{\sum_{i=1}^{n} k_{i 0}}{s}+\frac{D_{1} s^{3}+D_{2} s}{\left(s^{2}+\omega_{z 1}^{2}\right)\left(s^{2}+\omega_{z 2}^{2}\right)} \\
& +\cdots+\frac{C_{1} s^{2 n-6}+C_{2} s^{2 n-8}+\cdots+C_{0} s}{\left(s^{2}+\omega_{z 3}^{2}\right) \cdots\left(s^{2}+\omega_{z(n-1)}^{2}\right)} \\
\frac{Z_{U}(s)}{H}= & \frac{E_{1} s^{5}+E_{2} s^{3}+E_{0} s}{s\left(s^{2}+\omega_{z 1}^{2}\right)\left(s^{2}+\omega_{z 2}^{2}\right)}+\cdots \\
& +\frac{C_{1} s^{2 n-6}+C_{2} s^{2 n-8}+\cdots+C_{0} s}{s\left(s^{2}+\omega_{z 3}^{2}\right) \cdots\left(s^{2}+\omega_{z(n-1)}^{2}\right)},
\end{aligned}
$$

where $B_{1}, B_{2}, B_{0}, C_{1}, C_{2}, C_{0}, D_{1}, D_{2}, E_{1}, E_{2}, E_{0}$ are parameters determined by adding together the partial fractions obtained in (10).

The obtained rational functions, as the components of the sum of $(11) \div(14)$, are decomposed into continued fractions. In order to decompose into a continued fraction, for example, the function $\left(D_{1} s^{3}+D_{2} s\right) /\left(s^{2}+\omega_{z 1}^{2}\right)\left(s^{2}+\omega_{z 2}^{2}\right)$ from (13), obtained by impedance decomposition equation (4), the considered function, should be presented in the form of the quotient of two polynomials:

$$
\begin{aligned}
\frac{Z_{1}(s)}{H} & =\frac{D_{1} s^{3}+D_{2} s}{\left(s^{2}+\omega_{z 1}^{2}\right)\left(s^{2}+\omega_{z 2}^{2}\right)}=\frac{L_{1}(s)}{M_{1}(s)}=\frac{1}{\frac{M_{11}(s)}{L_{11}(s)}} \\
& =\frac{1}{Y_{1}(s)} .
\end{aligned}
$$

After dividing, in (15), the denominator by the numerator, we obtain

$$
\begin{aligned}
\frac{Z_{1}(s)}{H} & =\frac{1}{\frac{M_{11}(s)}{L_{11}(s)}}=\frac{1}{Y_{11}(s)+\frac{1}{Z_{11}(s)}} \\
& =\frac{1}{\frac{s}{c_{11}}+\frac{1}{\frac{L_{11}(s)}{M_{12}(s)}}}=\frac{1}{\frac{s}{c_{11}}+\frac{1}{Y_{11}(s)}},
\end{aligned}
$$

where $c_{11}$ is value of the determined spring component.

The next stage of decomposition of the function $Z_{1}(s) / H$ into a continued fraction is realization of the expression $Z_{11}(s)$ in (16). After dividing $L_{11}(s)$ by $M_{11}(s)$, we obtain

$$
\begin{aligned}
Z_{11}(s) & =m_{11} s+\frac{L_{12}(s)}{M_{12}(s)}=m_{11} s+\frac{1}{\frac{M_{12}(s)}{L_{12}(s)}} \\
& =m_{11} s+\frac{1}{Y_{12}(s)},
\end{aligned}
$$

where $m_{11}$ is value of the determined inertial component. 
The synthesized impedance $Z_{1}(s)$ takes the form

$$
\frac{Z_{1}(s)}{H}=\frac{1}{\frac{s}{c_{11}}+\frac{1}{m_{11} s+\frac{1}{Y_{12}(s)}}}
$$

The mobility $Y_{12}(s)$ in dependency equation (18) is realized as

$$
\begin{aligned}
Y_{12}(s) & =\frac{s}{c_{12}}+\frac{M_{13}(s)}{L_{12}(s)}=\frac{s}{c_{12}}+\frac{1}{\frac{L_{12}(s)}{M_{13}(s)}} \\
& =\frac{s}{c_{12}}+\frac{1}{Z_{12}(s)},
\end{aligned}
$$

where $c_{12}$ is value of the determined spring component.
After carrying out operations defined by dependency equation (19), the impedance $Z_{1}(s)$ is obtained in the form

$$
\frac{Z_{1}(s)}{H}=\frac{1}{\frac{s}{c_{11}}+\frac{1}{m_{11} s+\frac{1}{\frac{s}{c_{12}}+\frac{1}{Z_{12}(s)}}}}
$$

The process, described by dependencies equations (15) $\div$ (20), is continued until the moment when, as a result of polynomials dividing $(L(s), M(s)), c / s$ or $m s(c \in R \cap m \in R)$ is obtained.

Realizing the decomposition of all rational functions in (13), we determine the values required of inertial and elastic two-terminals in the case of the dynamic properties described in the form of impedance equation (4). Finally, the dynamic characteristics equation (4) subjected to the identification with the mixed method takes the general form of the sum of continued fractions in the form of

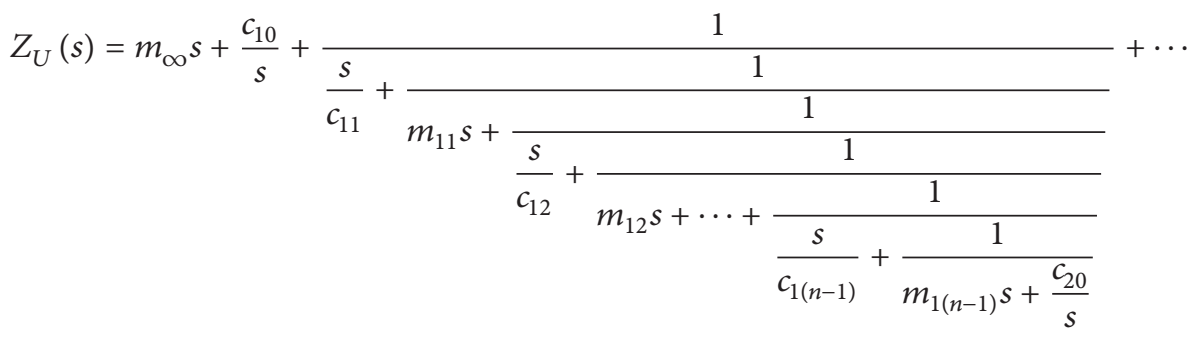

$$
\begin{aligned}
& 1
\end{aligned}
$$

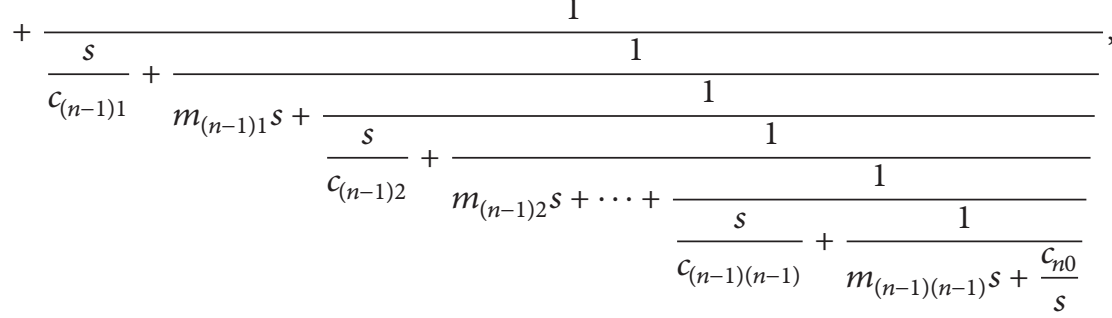

where $m_{\infty}, m_{11}, m_{1(n-1)}, m_{1 n}, m_{(n-1)(n-1)}$ are values of the inertial components of the sought system, $c_{10}, c_{20}, c_{n 0}, c_{(n+1) 0}$ are values of the springs restrained components, and $c_{11}, c_{1(n-1)}, c_{1 n}, c_{(n-1)(n-1)}$ are values of the springs components of the sought system.

Indexes (scripts) located at values of the designated parameters indicate, respectively, the number of branches and the number of two-terminals of the same type occurring in the bifurcation. The values of the elastic, restrained components are obtained by decomposition of the restraint rigidity $c_{0}=\sum_{k=1}^{n+1} c_{k 0}$ into the sum of components and multiplying by $c_{k 0} / s$ any rational function of the obtained sum (11) $\div(14)$. The number of components of the sum $c_{0}=\sum_{k=1}^{n+1} c_{k 0}$ depends on the restraints imposed on the sought system. The general form of the system structure obtained by the decomposition of impedance function equation (21) with the mixed method is shown in Figure 1.

\section{Active Vibration Reduction}

Inverse task [17, 24-26], presented in the work, not only solves the problem of the search for the structure and parameters (inertial, spring) of vibrating systems for the desired frequency spectrum, but also allows determining the components reducing vibration of the system to the desired amplitude value. Active vibration reduction with methods of the synthesis of vibrating systems refers to the systems whose dynamic properties and the forces reducing vibration are given in the analytical form.

3.1. Dynamic Properties of Active Vibration Reduction. Due to the required dynamic properties, which characterize the mode of action of the sought active force, four cases should be distinguished.

The first case occurs when the force reducing vibration dampens the selected resonance frequency of the dynamic 


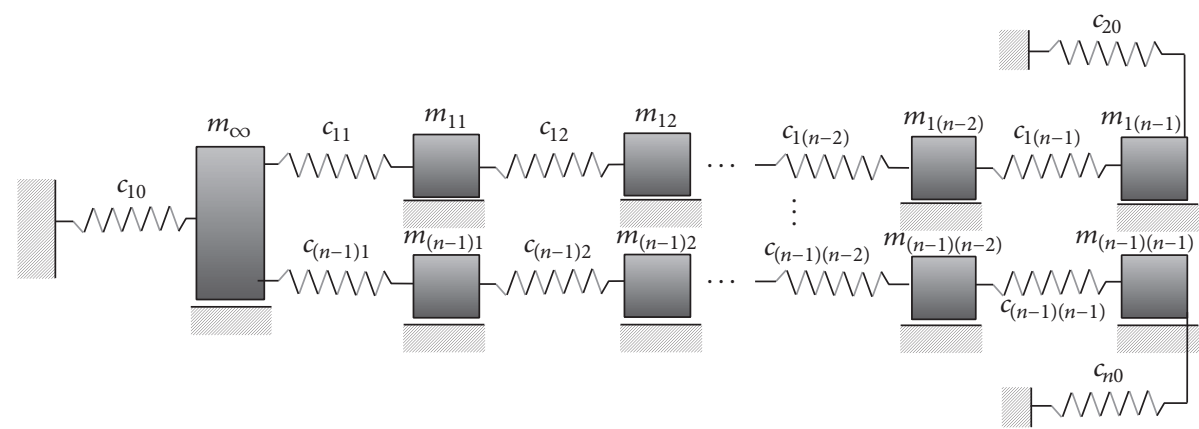

FIGURE 1: Cascade-branched structure as physical realization of impedance function equation (4) decomposition with the mixed method.

characteristics to the desired value of the amplitude of the deflection of the inertial component being under consideration. Dynamic properties are described by the impedance function in the form

$$
\begin{aligned}
& Z_{\mathrm{I}}(s)=\frac{1}{Y_{\mathrm{I}}(s)}=H \frac{\prod_{i=1}^{n}\left(s^{2}+2 h_{p i} s+\omega_{p i}^{2}+h_{p i}^{2}\right)}{s \prod_{j=1}^{n}\left(s^{2}+\omega_{z j}^{2}\right)}=H \\
& \cdot \frac{s \prod_{i=1}^{n}\left(s^{2}+\omega_{p i}^{2}\right)}{\prod_{j=1}^{n}\left(s^{2}+\omega_{z j}^{2}\right)}+H \\
& \cdot \frac{s \prod_{i=1}^{n}\left(s^{2}+2 h_{p i} s+\left(\omega_{p i}^{2}+h_{p i}^{2}\right)\right)-s \prod_{i=1}^{n}\left(s^{2}+\omega_{p i}^{2}\right)}{\prod_{j=1}^{n}\left(s^{2}+\omega_{z j}^{2}\right)} \\
& =H \frac{\prod_{i=1}^{n}\left(s^{2}+\omega_{p i}^{2}\right)}{s \prod_{j=1}^{n}\left(s^{2}+\omega_{z j}^{2}\right)}+\frac{L_{R}(s)}{M(s)}=Z_{U}(s)+Z_{R}(s),
\end{aligned}
$$

where $\omega_{p 1}, \omega_{p 2}, \ldots, \omega_{p i}(i=1,2,3, \ldots, n)$ are resonance frequencies, $\omega_{z 1}, \omega_{z 2}, \ldots, \omega_{z j}(j=1,2,3, \ldots, n)$ are antiresonance frequencies, $h_{p 1}, h_{p 2}, \ldots, h_{p i}(i=1,2,3, \ldots, n)$ are damping coefficient in the frequency units, $Z_{R}(s)$ is function characterizing the forces reducing vibration of the system, $H$ is proportionality constant, and $s$ is Laplace operator.

The second case occurs when the force reducing vibration acts as a dynamic vibration eliminator. Such force does not dampen the system but causes the system to leave the resonance zone, shifting the resonance frequency to any resonance zone chosen by the designer. The dynamic properties of sought system of vibration reduction (the impedance function) in this case are as follows:

$$
\begin{aligned}
Z_{\mathrm{II}}(s) & =\frac{1}{Y_{\mathrm{II}}(s)}=H \frac{\prod_{i=1}^{n}\left(s^{2}+\left(\omega_{p i}+\Delta \omega_{p i}\right)^{2}\right)}{s \prod_{j=1}^{n}\left(s^{2}+\omega_{z j}^{2}\right)} \\
& =H \frac{\prod_{i=1}^{n}\left(s^{2}+\omega_{p i}^{2}\right)}{s \prod_{j=1}^{n}\left(s^{2}+\omega_{z j}^{2}\right)}+\frac{L_{R}(s)}{M(s)} \\
& =Z_{U}(s)+Z_{R}(s),
\end{aligned}
$$

where $\Delta \omega_{p 1}, \Delta \omega_{p 2}, \ldots, \Delta \omega_{p i}(i=1,2,3, \ldots, n)$ are values of resonance frequencies shifts.

The third case occurs when the force reducing vibration dampens the desired resonance frequency to the amplitude value of deflection of the considered inertial component. In the system is a damped vibration frequency, in contrast to the first case, where the resonance frequency is the frequency of damped vibration of the system. The properties of the analyzed case should be selected as follows:

$$
\begin{aligned}
Z_{\mathrm{III}}(s) & =\frac{1}{Y_{\mathrm{III}}(s)}=H \frac{\prod_{i=1}^{n}\left(s^{2}+2 h_{p i} s+\omega_{p i}^{2}\right)}{s \prod_{j=1}^{n-1}\left(s^{2}+\omega_{z j}^{2}\right)} \\
& =H \frac{\prod_{i=1}^{n}\left(s^{2}+\omega_{p i}^{2}\right)}{s \prod_{j=1}^{n}\left(s^{2}+\omega_{z j}^{2}\right)}+\frac{L_{R}(s)}{M(s)} \\
& =Z_{U}(s)+Z_{R}(s) .
\end{aligned}
$$

The fourth case occurs when the force reducing vibration not only causes the system to leave the resonance zone, shifting the resonance frequency to any resonance zone, but also dampens the chosen zone to the required amplitude value of deflection of the considered inertial component. The dynamic properties are then as follows:

$$
\begin{aligned}
& Z_{\mathrm{IV}}(s)=\frac{1}{Y_{\mathrm{IV}}(s)}=H \\
& \cdot \frac{\prod_{i=1}^{n}\left(s^{2}+2 h_{p i} s+\omega_{p i}^{2}+2 \omega_{p i} \Delta \omega_{p i}+\Delta \omega_{p i}^{2}+h_{p i}^{2}\right)}{s \prod_{j=1}^{n}\left(s^{2}+\omega_{z j}^{2}\right)} \\
&=H \frac{\prod_{i=1}^{n}\left(s^{2}+\omega_{p i}^{2}\right)}{s \prod_{j=1}^{n}\left(s^{2}+\omega_{z j}^{2}\right)}+\frac{L_{R}(s)}{M(s)}=Z_{U}(s)+Z_{R}(s) .
\end{aligned}
$$

To be able to speak about the desired dynamic properties for vibration reduction, one should specify the parameters values of the dynamic characteristics subjected to the synthesis. The parameter which characterizes the analyzed systems of vibration reduction is the damping coefficient $h_{p i}$. Whereas the value determining the sought system of vibration reduction is the maximal amplitude, that should be achieved by the system during the occurrence in the system, the phenomenon of, for example, resonance. In this case, the dependence of the damping coefficient on the desired amplitude of vibration should be determined.

The desired amplitude of vibration is defined, in the work, as the maximal deflection of the inertial component 


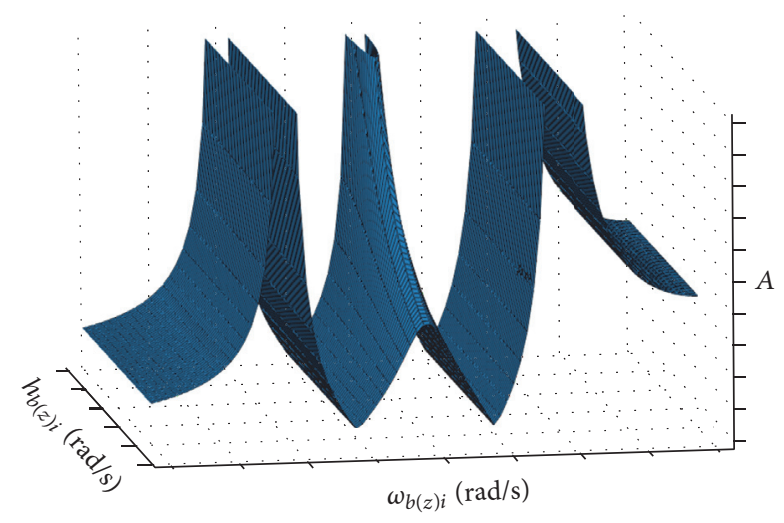

FIGURE 2: Graph of admittance $V(s)$.

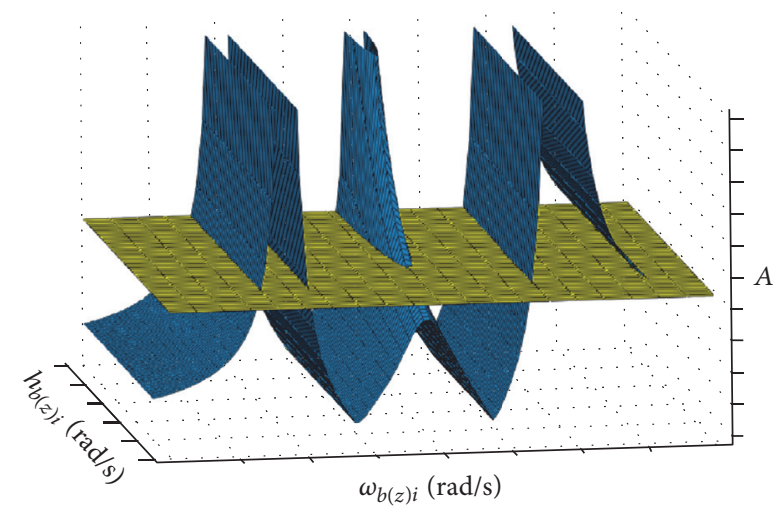

FIgURE 3: Characteristic function intersected by the plane $V_{A i}(s, h)=A_{p i}$.

corresponding to the system response at the unit amplitude of exciting force $(H=1)$. If the value of such amplitude is known, then, using the graph of dynamic characteristics, as a function of two variables, the frequency one $\left(\omega_{z j, p i}\right)$ and the damping coefficient $\left(h_{z j, p i}\right), h_{p i}$ is determined corresponding to the required amplitude for the selected resonant frequency $\omega_{p i}$. Depending on the amplitude of the vibration of the desired kinematic quantity, the graphs of admittance, mobility, or virtual mass are considered.

Let us give the maximal deflection $A_{\max }$, for the first case, which could be achieved by the inertial component with respect to which it is determined by the dynamic characteristics in the form of the impedance or mobility functions. In order to determine the coefficient $h_{p i}$, the graph of the admittance $V(s)=(1 / s) Y(s)=1 / s Z(s)$ should be generated as a function of two variables, which are the frequencies $\omega_{p i}$ and the decreasing coefficient $h_{p i}$.

In Figure 2 is shown the change of amplitude of the admittance function in the selected resonance zone.
Knowing the value of the amplitude $A_{p i}$ of the selected resonance zone, one should determine the following equation of a plane:

$$
V_{A i}(s, h)=A_{p i} \text {. }
$$

The determined plane intersects the admittance characteristics in the value of the chosen amplitude $A_{p i}$ (Figure 3).

Making the projection of the plotted area on the plane $(s, h)$, component $h$ of the maximal point specified by the plane $V_{A}\left(\omega_{p}, h\right)=A_{p}$ in the selected resonance zone is determined. The resultant component $h$ corresponds to the sought value of the free vibration frequency decrease coefficient.

Using the methods of active vibration reduction, it is possible to assume any value of the amplitude, in the case of successively chosen resonance frequencies, without affecting the previously determined coefficient of free vibration frequency decreasing. Further cases of determining the coefficient $h$, depending on the type of the sought force, are determined according to the presented method. The free vibration frequency decrease coefficient $h_{p}$ corresponding to the desired amplitude $A_{p}$ of the analyzed point of the system is the solution of the following system:

$$
\begin{aligned}
& V(s, h)=\frac{1}{s} Z(s, h)=H \\
& \quad \cdot \frac{\prod_{j=1}^{n-1}\left(s^{2}+\omega_{z j}^{2}\right)}{\left(s^{2}+2 h_{p} s+\omega_{p}^{2}+h_{p}^{2}\right) \prod_{i=1}^{n-1}\left(s^{2}+2 h_{p i} s+\omega_{p i}^{2}+h_{p i}^{2}\right)}, \\
& s=\omega_{p}, \\
& V_{A}(s, h)=A_{p} .
\end{aligned}
$$

3.2. Method of the Synthesis of Active Vibration Reduction to the Desired Amplitude Value. The function of the impedance $Z(s)$ (the inverse mobility) is the basis for conducting the identification and active vibration reduction using the methods of synthesis. As a result of decomposition of the dynamic characteristics, one receives two rational functions: $Z_{U}(s)$ and $Z_{R}(s)$. The function $Z_{R}(s)$ defines the conditions which must be satisfied by the sought active force. However, the function $Z_{U}(s)$ is the impedance function of the system being identified in equations $(22) \div(25)$. The active vibration reduction is carried out in two stages. In the first stage, parameters of the system subjected to reduction are designated, on the basis of a sequence of resonance antiresonance frequencies (Figure 5). The resulting structure and the parameters of the identified system are the starting point for applying the methods of active vibration reduction.

\section{Stage I. See Figure 5.}

Stage II. It is the force acting on the inertial component for which the impedance is determined as a result of the second stage of the active vibration reduction, which is equivalent to the impedance $Z_{U}(s)$ subjected to decomposition. For this purpose, the stiffness matrix of the system obtained as a result of utilization of the synthesis with the mixed method is determined. In general, the stiffness matrix becomes 


$$
\left[\begin{array}{ccccc}
m_{\infty} s^{2}+c_{11}+c_{10} & -\left(c_{11}\right) & \cdots & 0 & 0 \\
-\left(c_{11}\right) & m_{11} s^{2}+c_{1}+c_{11}+c_{12} & \cdots & 0 & 0 \\
\vdots & \vdots & \cdots & \vdots & \vdots \\
0 & 0 & \cdots & m_{1(n-2)} s^{2}+c_{(n-1)}+c_{1(n-2)}+c_{1(n-1)} & -\left(c_{1(n-1)}\right) \\
0 & 0 & \cdots & -\left(c_{1(n-1)}\right) & m_{1(n-1)} s^{2}+c_{1(n-1)}+c_{20}
\end{array}\right]=\mathbf{K}(s)
$$

The resulting matrix is the square one and its determinant $\operatorname{det} \mathbf{K}(s)=0$ is the characteristic equation of the identified system (Figure 5). As a result of expansion of the determinant of matrix equation (28), an algebraic equation of the degree $2(n+1)$ is obtained with respect to the unknown $s$. This equation has $2(n+1)$ roots, which are complex conjugated numbers and whose values are equal to the predetermined resonance frequencies.

Using the stiffness matrix, one should determine the algebraic complement $M_{x(n+1)}(s)$, which takes the form

$$
\begin{gathered}
M_{x 1}(s)=D_{11}(\mathbf{K})=(-1)^{1+1}\left|K_{11}\right| \\
M_{x 2}(s)=D_{12}(\mathbf{K})=(-1)^{1+2}\left|K_{12}\right| \\
M_{x 3}(s)=D_{13}(\mathbf{K})=(-1)^{1+3}\left|K_{13}\right| \\
\vdots \\
M_{x(n+1)}(s)=D_{1(n+1)}(\mathbf{K})=(-1)^{1+(n+1)}\left|K_{1(n+1)}\right| .
\end{gathered}
$$

For such determined complements, one proceeds with decomposition of the function $Z_{R}(s)$. The function $Z_{R}(s)=$ $L_{R}(s) / M(s)$ is denoted in the form of a quotient of two polynomials, whose numerator is multiplied by the Laplace operator and $\prod_{i=1}^{n-1} m_{1 i}$, as follows:

$$
Z_{R}(s)=\frac{L_{R}(s)}{M(s)} \Longrightarrow \frac{s L_{R}(s) \prod_{i=1}^{n-1} m_{1 i}}{M(s)}=\frac{L_{F 1}(s)}{M_{x 1}(s)},
$$

where $M_{x 1}(s)$ is the algebraic complement of stiffness matrix equation (28).

After dividing in (30) the numerator by the denominator, we obtain

$$
\frac{L_{F 1}(s)}{M_{x 1}(s)}=F_{x 1}(s)+\frac{L_{F 2}(s)}{M_{x 1}(s)}=a_{1} s+b_{1}+\frac{L_{F 2}(s)}{M_{x 1}(s)},
$$

where the designated parameters are components of the sought active force.

In the next step, the numerator obtained from (31) should be divided by the matrix determinant, designated in (29):

$$
\frac{L_{F 2}(s)}{M_{x 2}(s)}=F_{x 2}(s)+\frac{L_{F 3}(s)}{M_{x 2}(s)}=a_{2} s+b_{2}+\frac{L_{F 3}(s)}{M_{x 2}(s)} \text {. }
$$

On the basis of the function $L_{F 3}(s)$, the successive components of the force $F(s)$ are determined in the next way:

$$
\frac{L_{F 3}(s)}{M_{x 3}(s)}=F_{x 3}(s)+\frac{L_{F 4}(s)}{M_{x 3}(s)}=a_{3} s+b_{3}+\frac{L_{F 4}(s)}{M_{x 3}(s)} \text {. }
$$

Dividing process is continued until the remainder is not obtained after dividing the numerator by the denominator, which is noted as follows:

$$
\frac{L_{F n}(s)}{M_{x n}(s)}=F_{x n}(s)=a_{n} s+b_{n}
$$

Finally the components of the force take the next form:

$$
\begin{aligned}
& F(s)=-\left(a_{1} s+b_{1}+a_{2} s+b_{2}+a_{3} s+b_{3} \cdots+a_{n} s+b_{n}\right) \Longrightarrow \\
& F(\dot{x}, x, t)=-\left(a_{1} \dot{x}_{1}(t)+b_{1} x_{1}(t)+a_{2} \dot{x}_{2}(t)+b_{2} x_{2}(t)\right. \\
& \left.\quad+\cdots+a_{n} \dot{x}_{n}(t)+b_{n} x_{n}(t)\right),
\end{aligned}
$$

where $a_{1}, a_{2}, b_{1}, b_{2}, \ldots, a_{n}, b_{n}$ are values determined during the conducted synthesis, $x_{1}(t), x_{2}(t), \ldots, x_{n}(t)$ are generalized coordinates of the adequate inertia components, and $\dot{x}_{1}(t), \dot{x}_{2}(t), \ldots, \dot{x}_{n}(t)$ are generalized velocities of the particular inertia components.

As a result of the conducted synthesis of systems with active vibration reduction, the system is obtained (Figure 6), whose structure and values of determined parameters meet the required dynamic properties.

3.3. Numerical Example. Let us give the dynamic properties, in the form of resonance and antiresonance frequencies, and the amplitude value of the sought system:

$$
\begin{aligned}
& \omega_{p 1}=121 \mathrm{rad} / \mathrm{s}, \\
& \omega_{p 2}=175 \mathrm{rad} / \mathrm{s}, \\
& \omega_{p 3}=330 \mathrm{rad} / \mathrm{s} \\
& \omega_{z 1}=148 \mathrm{rad} / \mathrm{s}, \\
& \omega_{z 2}=252 \mathrm{rad} / \mathrm{s} \\
& A_{p 3}=0,0003 \mathrm{~m} .
\end{aligned}
$$

It is assumed that the structure of the sought system is in the form of a cascade model shown in Figure 7. 

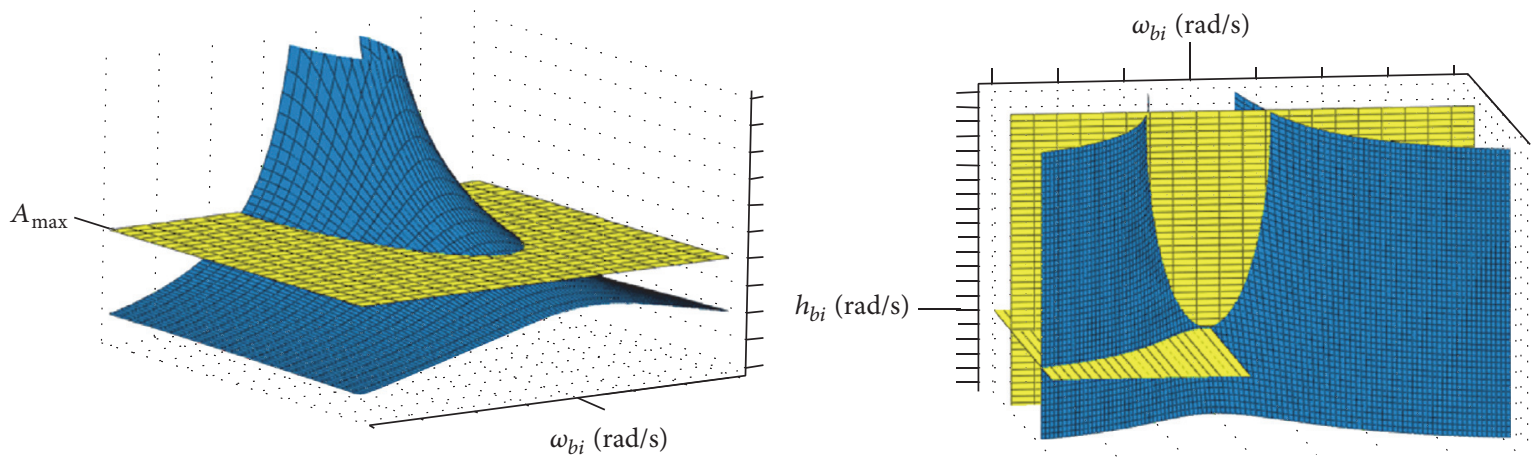

FIGURE 4: Graphical interpretation of the method for damping coefficient determination.

The analytic form of the dynamic characteristics describing the desired dynamic properties, in the case of active vibration reduction, takes the following form of the impedance:

$$
\begin{aligned}
& Z(s) \\
& =\frac{\left(s^{2}+121^{2}\right)\left(s^{2}+175^{2}\right)\left(s^{2}+2 h_{b 3} s+330^{2}+h_{b 3}^{2}\right)}{s\left(s^{2}+148^{2}\right)\left(s^{2}+252^{2}\right)},
\end{aligned}
$$

$$
\text { for } H=1 \text {. }
$$

On the basis of such adopted dynamic characteristics equation (37), the graph of admittance $V(s, h)$ and the plane corresponding to the desired amplitude $A_{p 3}=0,0003 \mathrm{~m}$ is determined. The set of points obtained by the intersection of the two graphs (see Figure 4) determines the appropriate indication of the value of the sought decrease coefficient $h_{p 3}$ :

$$
\begin{aligned}
& V(s, h)=\frac{1}{s Z(s, h)} \\
& =\left.\frac{\left(s^{2}+148^{2}\right)\left(s^{2}+252^{2}\right)}{\left(s^{2}+121^{2}\right)\left(s^{2}+175^{2}\right)\left(s^{2}+2 h_{p 3} s+330^{2}+h_{p 3}^{2}\right)}\right|_{s=\omega_{p 3}} \\
& V_{A}(s, h)=A_{p 3} .
\end{aligned}
$$

The determined damping coefficient takes the following value:

$$
h_{p 3}=2,7 \mathrm{rad} / \mathrm{s}
$$

Including (39) in (37), one obtains the impedance function in the form

$$
\begin{aligned}
Z(s)= & \frac{\left(s^{2}+121^{2}\right)\left(s^{2}+175^{2}\right)\left(s^{2}+5,4 s+332,7^{2}\right)}{s\left(s^{2}+148^{2}\right)\left(s^{2}+252^{2}\right)}, \\
Z(s)= & Z_{U}(s)+Z_{R}(s) \\
= & \frac{\left(s^{2}+121^{2}\right)\left(s^{2}+175^{2}\right)\left(s^{2}+330^{2}\right)}{s\left(s^{2}+148^{2}\right)\left(s^{2}+252^{2}\right)} \\
& +\frac{\left(s^{2}+121^{2}\right)\left(s^{2}+175^{2}\right)\left(5,4 s+2,7^{2}\right)}{s\left(s^{2}+148^{2}\right)\left(s^{2}+252^{2}\right)} .
\end{aligned}
$$

As a result of decomposition of the function $Z_{U}(s)$ with the mixed method of the synthesis, parameters equation (41) of the identified system is obtained:

$$
\begin{aligned}
Z_{U}(s)= & m_{\infty} s+\frac{c_{10}}{s} \\
+ & \frac{1}{\frac{s}{c_{11}}+\frac{1}{m_{11} s+\frac{1}{\frac{s}{c_{12}}+\frac{1}{m_{12} s+\frac{c_{20}}{s}}}}},
\end{aligned}
$$

where $m_{\infty}=1,000 \mathrm{~kg}, m_{11}=1,39 \mathrm{~kg}, m_{12}=6,944 \mathrm{~kg}, c_{11}=$ $51206,26 \mathrm{~N} / \mathrm{m}, c_{12}=33036,34 \mathrm{~N} / \mathrm{m}, c_{10}=17551,74 \mathrm{~N} / \mathrm{m}$, and $c_{20}=139356,47 \mathrm{~N} / \mathrm{m}$.

In the next step the values of the active force (Figure 7) are determined, acting on the inertial component $m_{\infty}$, in respect to which the system response is generated. To realize this one should determine the stiffness matrix of the resulting system (Figure 7):

$$
\begin{aligned}
& \mathbf{K}(s) \\
& =\left[\begin{array}{ccc}
m_{\infty} s^{2}+c_{11}+c_{10} & -\left(c_{11}\right) & 0 \\
-\left(c_{11}\right) & m_{11} s^{2}+c_{1}+c_{11}+c_{12} & -\left(c_{12}\right) \\
0 & -\left(c_{12}\right) & m_{12} s^{2}+c_{12}+c_{20}
\end{array}\right] .
\end{aligned}
$$

Based on resulting stiffness matrix equation (42), the algebraic complement in accordance with the presented equations is determined:

$$
\begin{aligned}
& M_{x 1}(s)=D_{11}(\mathbf{K})=(-1)^{1+1}\left|K_{11}\right| \\
& M_{x 2}(s)=D_{12}(\mathbf{K})=(-1)^{1+2}\left|K_{12}\right| \\
& M_{x 3}(s)=D_{13}(\mathbf{K})=(-1)^{1+3}\left|K_{13}\right| .
\end{aligned}
$$




$$
Z_{U}(s)=m_{\infty} s+\frac{c_{10}}{s}+\frac{\omega_{p 1}, \omega_{p 2}, \ldots, \omega_{p i}}{s}+\frac{\omega_{z 1}, \omega_{z 2}, \ldots, \omega_{z j}}{c_{11}}+\frac{{ }_{u} c_{1(1)}}{s}+m_{11} s+\frac{s \prod_{j=1}^{n-1}\left(s^{2}+\omega_{z j}^{2}\right)}{\prod_{i=1}^{n}\left(s^{2}+\omega_{p i}^{2}\right)}
$$

FIGURE 5: Physical implementation of dynamical characteristics in the form of a continued fraction sum.

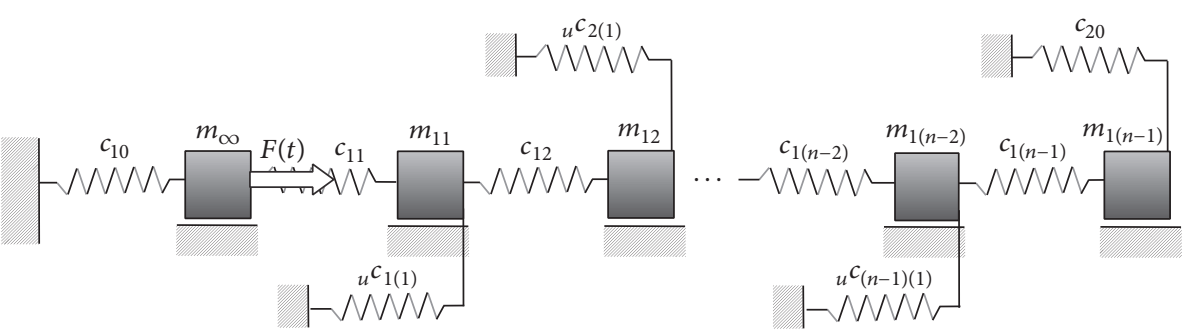

FIGURE 6: Structure of the system searched including the active damping force.

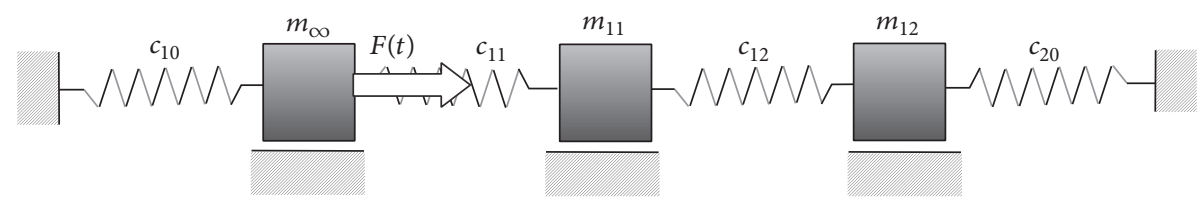

FIGURE 7: Model of structure of the sought system including the active damping force. 


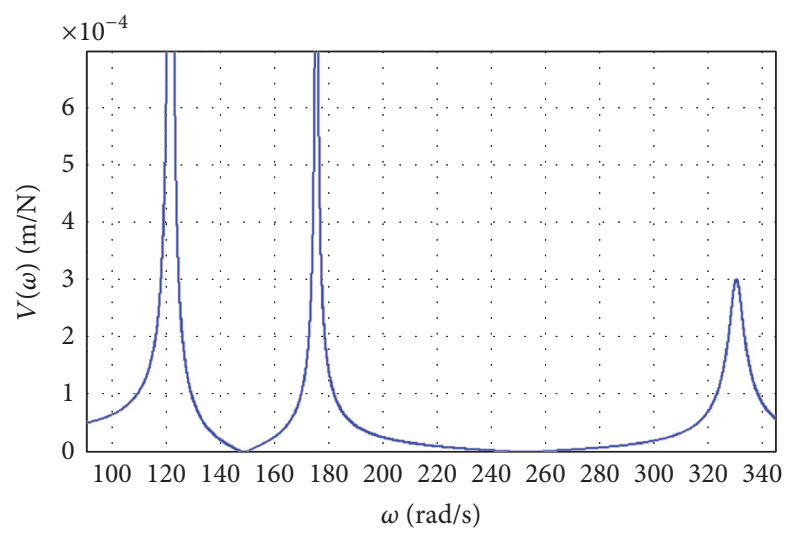

Figure 8: Dynamical characteristics for the admittance of systems obtained as a result of active vibration reduction performed by using the direct method.
The rational function $Z_{R}(s)$, in the form of a quotient of two polynomials, should be presented in the following form:

$$
\begin{aligned}
& Z_{R}(s)=\frac{L_{R}(s)}{M_{x 1}(s)} \Longrightarrow \\
& \frac{\prod_{i=1}^{n-1} m_{1 i} s L_{R}(s)}{M_{x 1}(s)} \\
& =\frac{\left(s^{2}+121^{2}\right)\left(s^{2}+175^{2}\right)\left(5,4 s+2,7^{2}\right)}{\left(s^{2}+148^{2}\right)\left(s^{2}+252^{2}\right)},
\end{aligned}
$$

where $M_{x 1}(s)$ is algebraic complement of stiffness matrix equation (42).

After dividing in (44) the numerator by the denominator, we obtain

$$
\frac{L_{F 1}(s)}{M_{x 1}(s)}=F_{x 1}(s)+\frac{L_{F 2}(s)}{M_{x 1}(s)}=5,4 s+7,29+\frac{-216766,8 s^{3}-292635,2 s^{2}-5090099351,4 s-6871634124,4}{\left(s^{2}+148^{2}\right)\left(s^{2}+252^{2}\right)}
$$

The numerator $L_{F 2}(s)$ is divided by the determinant of the matrix $M_{x 2}(s)$, in accordance with (45), obtaining the parameters of force equation (46) that depended on the generalized coordinate associated with the inertial component $m_{11}$ :

$$
\begin{aligned}
& \frac{L_{F 2}(s)}{M_{x 2}(s)} \\
& =\frac{-216766,8 s^{3}-292635,2 s^{2}-5090099351,4 s-6871634124,4}{36839 s^{2}+914571562,3} \\
& =F_{x 2}(s)+\frac{L_{F 3}(s)}{M_{x 2}(s)} \\
& =-5,88 s-7,943+\frac{291391756,7 s+393378871,5}{36839 s^{2}+914571562,3} .
\end{aligned}
$$

Dividing, in the further step, $L_{F 3}(s)$ from (46) by $M_{x 3}(s)$, the components of the force $F(s)$, depending on the displacement of the inertial component, are determined obtaining

$$
\begin{aligned}
& \frac{291391756,7 s+393378871,5}{175263092,9}=F_{x 3}(s) \\
& =1,66 s+2,44 .
\end{aligned}
$$

As a result of the decomposition of the function $Z_{F}(s)$, the obtained force takes the form

$$
\begin{aligned}
& F(s)=-(5,4 s+7,29-5,88 s-7,943+1,66 s+2,44) \Longrightarrow \\
& F(\dot{x}, x, t)=-\left(5,4 \dot{x}_{1}(t)+7,29 x_{1}(t)-5,88 \dot{x}_{2}(t)\right. \\
& \left.\quad-7,943 x_{2}(t)+1,66 \dot{x}_{2}(t)+2,44 x_{2}(t)\right) .
\end{aligned}
$$

As the method for verification of the solutions obtained as a result of vibration reduction, the characteristics of the system admittance have been determined (Figure 7). It was assumed that on the system acts unit excitation force with the frequency equal to the reduced frequency. The generated dynamic characteristic (Figure 8) confirms the correctness of carried out vibration reduction.

\section{Conclusions}

The synthesis methods, presented in authors' works, and their formulation and formalization allow finding a mechanical system and adopting its nature of functioning and then determining the values of the actuating force. The value of this force should be such determined that the movement of this system would be consistent with the requirements assumed with regard to the characteristic function. The positive solution of this problem allows conducting qualitatively new research as well as obtaining new solutions and generalizations that are difficult to predict with the previously used methods of description and designing the systems with specific dynamic properties. The formalism, presented in works, forms the basis for the development of software systems designated for the passive and active synthesis of mechanical systems. The paper presents also the conditions for physical realization of the dynamic characteristics that describe the dynamic properties of the sought structure. The designated control forces may be the measure of structural sensitiveness of systems with characteristics that meet the same dynamic properties. Such approach is caused by the variety of values of forces obtained depending on the system subjected to active vibration reduction. It is possible to conclude that the smaller the value of the control force, the greater the structural sensitiveness of the system to the change of the reduced resonance frequency. Moreover, the set of values of meeting the same dynamic properties could be used to optimize the system control forces because of the desired vibration amplitude. 


\section{Competing Interests}

The authors declare that they have no competing interests.

\section{References}

[1] M. Z. Kolovsky, Nonlinear Dynamics of Active and Passive Systems of Vibration Protection, Foundations of Engineering Mechanics, Springer, Berline, Germany, 1999.

[2] A. Dymarek and T. Dzitkowski, "Active synthesis of discrete systems as a tool for reduction vibration," Solid State Phenomena, vol. 198, pp. 427-432, 2013.

[3] S.-M. Kim, S. Wang, and M. J. Brennan, "Dynamic analysis and optimal design of a passive and an active piezo-electrical dynamic vibration absorber," Journal of Sound and Vibration, vol. 330, no. 4, pp. 603-614, 2011.

[4] J. E. Mottershead and Y. M. Ram, "Inverse eigenvalue problems in vibration absorption: passive modification and active control," Mechanical Systems and Signal Processing, vol. 20, no. 1, pp. 5-44, 2006.

[5] M. Płaczek, "Modelling and investigation of a piezo composite actuator application," International Journal of Materials and Product Technology, vol. 50, no. 3-4, pp. 244-258, 2015.

[6] T. Dzitkowski and A. Dymarek, "Active reduction of identified machine drive system vibrations in the form of multi-stage gear units," Mechanika, vol. 20, no. 2, pp. 183-189, 2014.

[7] M. A. Louroza, N. Roitman, and C. Magluta, "Vibration reduction using passive absorption system with Coulomb damping," Mechanical Systems and Signal Processing, vol. 19, no. 3, pp. 537549, 2005.

[8] A. Dymarek and T. Dzitkowski, "Passive reduction in vibration considering the desired amplitude in the case of damping proportional to the mass," Solid State Phenomena, vol. 220-221, pp. 43-48, 2015.

[9] A. Dymarek and T. Dzitkowski, "Passive reduction of system vibrations to the desired amplitude value," Journal of Vibroengineering, vol. 15, no. 3, pp. 1354-1364, 2013.

[10] M. C. Smith, "Synthesis of mechanical networks: the inerter," IEEE Transactions on Automatic Control, vol. 47, no. 10, pp. 1648-1662, 2002.

[11] A. Gwiazda, "Construction development using virtual analysis on the example of a roof support," Applied Mechanics and Materials, vol. 474, pp. 417-422, 2014.

[12] M. P. Hetmańczyk, "The multilevel prognosis system based on matrices and digraphs methods," Solid State Phenomena, vol. 199, pp. 79-84, 2013.

[13] A. Sękala and J. Świder, "Hybrid graphs in modelling and analysis of discrete-continuous mechanical systems," Journal of Materials Processing Technology, vol. 164-165, pp. 1436-1443, 2005.

[14] R. C. Redfield and S. Krishnan, "Dynamic system synthesis with a bond graph approach. Part I-synthesis of one-port impedances," Journal of Dynamic Systems, Measurement and Control, vol. 115, no. 3, pp. 357-363, 1993.

[15] R. C. Redfield, "Dynamic system synthesis with a bond graph approach, part II: conceptual design of an inertial velocity indicator," Journal of Dynamic Systems, Measurement, and Control, vol. 115, no. 3, pp. 364-369, 1993.

[16] J. S. Park and J. S. Kim, "Dynamic system synthesis in term of bond graph prototypes," KSME International Journal, vol. 12, no. 3, pp. 429-440, 1998.
[17] A. Dymarek and T. Dzitkowski, "The inverse problem of kinematics as the tool for determination of motion trajectories exercised by worm drives of a Stewart platform," Advanced Materials Research, vol. 837, pp. 357-362, 2014.

[18] E. Świtoński and A. Mężyk, "Selection of optimum dynamic features for mechatronic drive systems," Automation in Construction, vol. 17, no. 3, pp. 251-256, 2008.

[19] A. Buchacz, A. Dymarek, and T. Dzitkowski, "Design and examining of sensitivity of continuous and discrete-continuous mechanical systems with required frequency spectrum represented by graphs and structural numbers," Monograph 88 , Silesian University of Technology Press, Gliwice, Poland, 2005 (Polish).

[20] A. Dymarek, Active and Passive Vibration Reduction in Mechanical Systems Depending on the Synthesis Method, Monograph no. 480, Silesian University of Technology Press, Gliwice, Poland, 2013 (Polish).

[21] K. Bialas and A. Sękala, "Vibration analysis of mechanical systems with the discrete-continuous distribution of parameters," Solid State Phenomena, vol. 198, pp. 698-703, 2013.

[22] M. Płaczek, "Dynamic characteristics of a piezoelectric transducer with structural damping," Solid State Phenomena, vol. 198, pp. 633-638, 2013.

[23] D. J. Inman, Vibration with Control, John Wiley \& Sons, New York, NY, USA, 2006.

[24] G. C. Teme and S. K. Mitra, Eds., Modern Filter Theory and Design, John Wiley and Sons, New York, NY, USA, 1973.

[25] T. Dzitkowski and A. Dymarek, "Method of active and passive vibration reduction of synthesized bifurcated drive systems of machines to the required values of amplitudes," Journal of Vibroengineering, vol. 17, no. 4, pp. 1578-1592, 2015.

[26] T. Dzitkowski and A. Dymarek, "Active synthesis of discrete systems as a tool for stabilisation vibration," Applied Mechanics and Materials, vol. 307, pp. 295-298, 2013. 


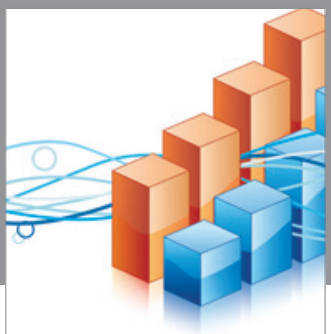

Advances in

Operations Research

vatem alat4

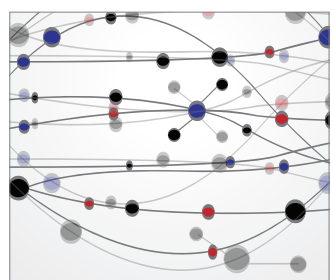

\section{The Scientific} World Journal
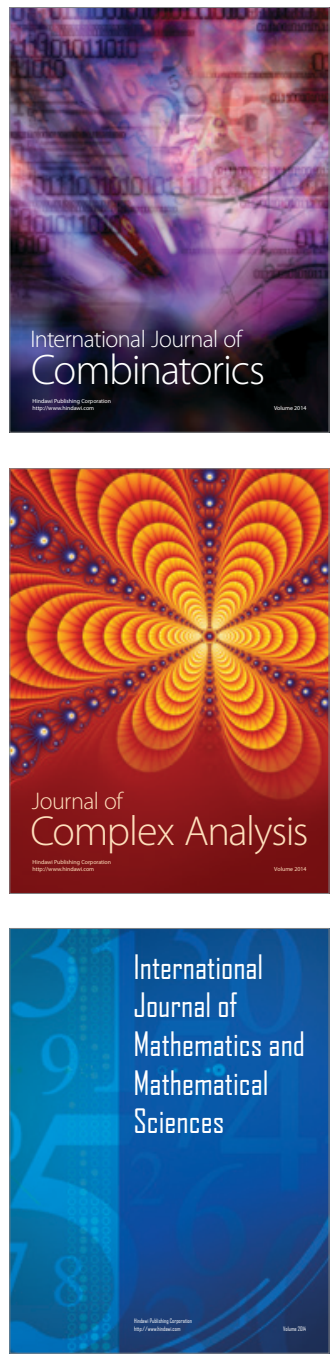
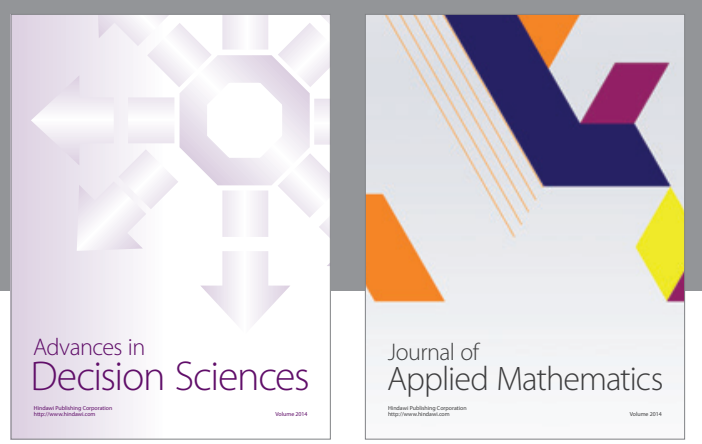

Algebra

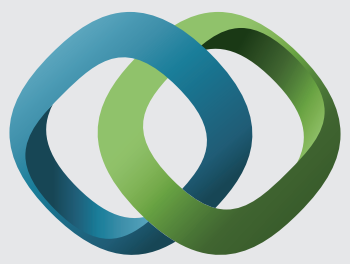

\section{Hindawi}

Submit your manuscripts at

http://www.hindawi.com
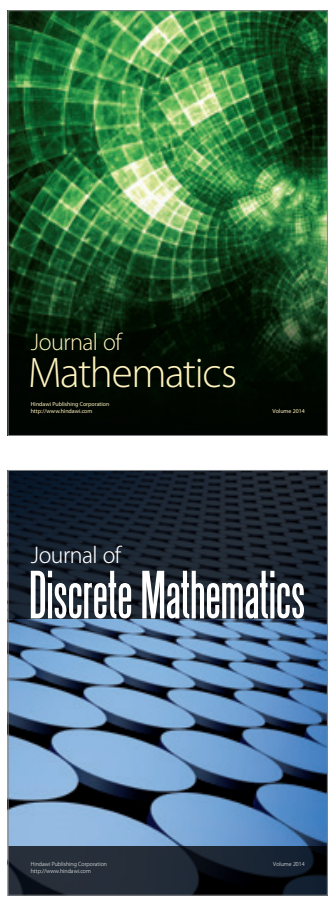

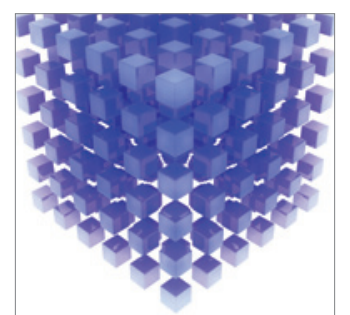

Mathematical Problems in Engineering
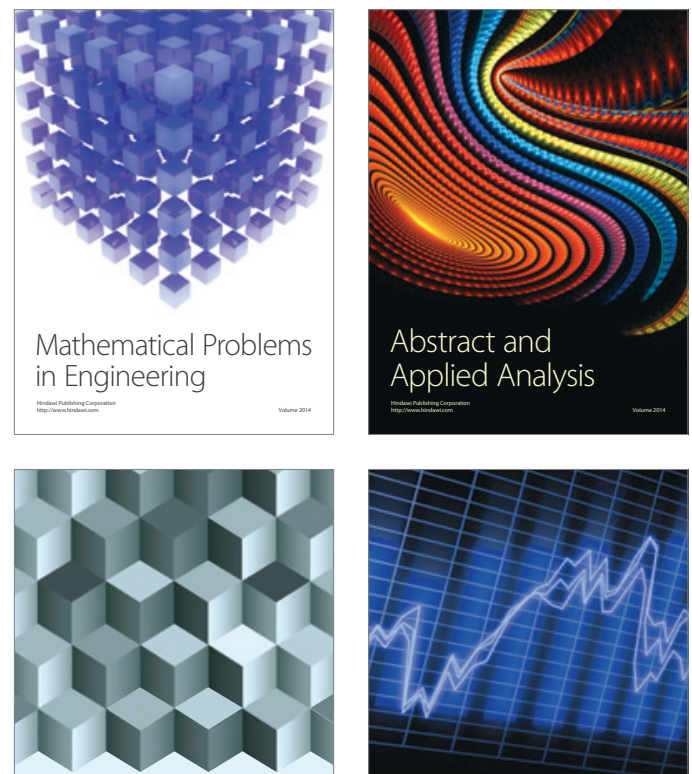

Journal of

Function Spaces

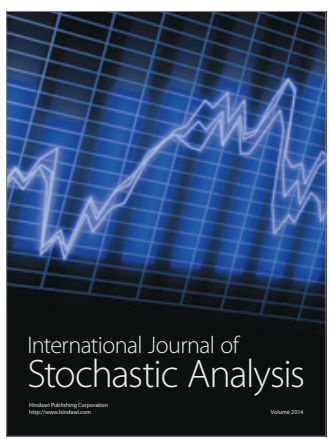

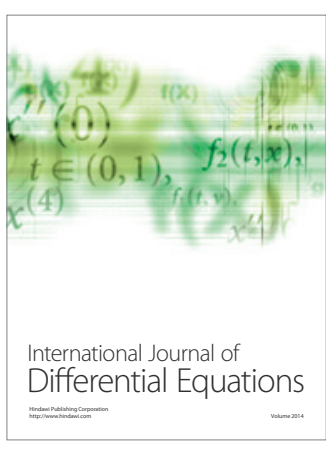
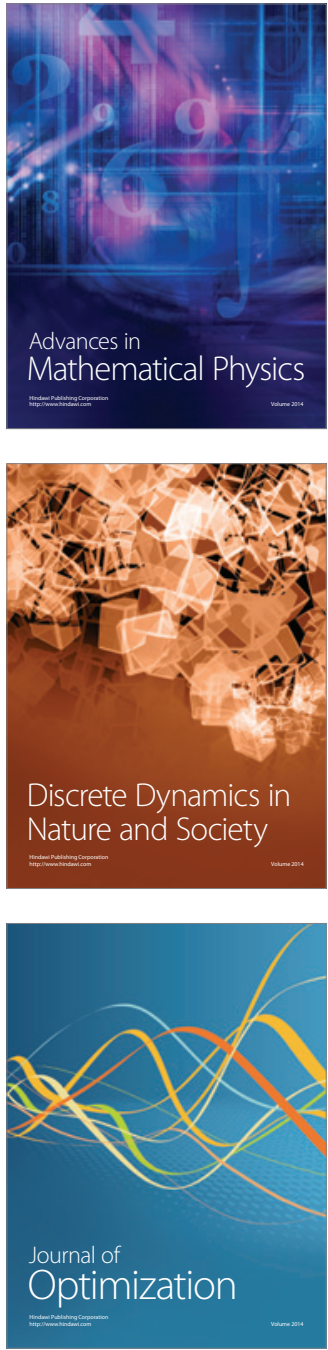